\title{
Standardized Process as a Tool for Higher Level Systems Thinking
}

\author{
Caroline Twomey Lamb \\ cmtwomey@mit.edu \\ Massachusetts Institute of Technology \\ 41-205 \\ 77 Massachusetts Avenue \\ Cambridge, MA 02139
}

\author{
Donna H. Rhodes \\ rhodes@mit.edu \\ Massachusetts Institute of Technology \\ NE20-388 \\ 77 Massachusetts Avenue \\ Cambridge MA, 02139
}

Copyright $\odot 2007$ by Caroline Lamb and Donna Rhodes. Published and used by INCOSE with permission.

\begin{abstract}
Standardized processes are being used to address three issues in engineering today: increasing system complexity; shifting demographics; and longer development cycles that result in a dearth of experiential learning for young engineers. There is obvious value in having engineers who can see the big picture and effectively broker design tradeoffs: engineers capable of systems thinking. However, what is the impact of standardized processes on the ability of teams of engineers to make these critical tradeoffs? This paper lays the groundwork for research investigating ways in which standardized process usage facilitates or hinders systems thinking within engineering teams-termed collaborative systems thinking.
\end{abstract}

\section{Introduction}

Systems engineering grew out of the increasing complexity of aerospace and defense products during and following World War II. (Johnson, 1997) As systems engineering has matured, standardized processes have emerged as a means to systemize the practice and deal with the unceasing increase in complexity of products designed and integrated. Much like specifying subsystems and defining interfaces, standardized processes are a way to break large pieces of work into smaller pieces and specify what bits of information need to be shared and with whom. Standardized processes help to reduce ambiguity and unpredictability. (Schein, 2004) These processes also attempt to ensure the efficient use of all resources: human, financial, and material.

Increasingly, standardized processes are also being looked to as a way to institutionalize design knowledge as engineers in many fields are retiring faster than new graduates are replacing them. The aerospace industry exemplifies this 'brain drain.' Employment in the industry dropped by nearly 50\% from 1989 to $2003^{1}$. Consequently fewer young graduates are seeking aerospace jobs. The result is a rising average within organizations such as NASA, where the current average age of an engineer is 49 (Lemos, 2006). As these experienced engineers prepare to retire en masse, less experienced engineers are left to design increasingly complex systems. The problem is these engineers have not benefited from repeated opportunities to exercise the design process in all of the life cycle phases since the number of available programs on which to do so has dramatically declined.

Systems thinking is best learned experientially, leaving engineers with less experience and fewer opportunities to gain experience at a disadvantage. However, standardized processes may

\footnotetext{
${ }^{1}$ Based on Aerospace Industries Association data <http://www.aia-aerospace.org/stats/aero_stats/stat12.pdf >
} 
be a tool, when aligned with organizational culture, to enforce and develop systems thinking. As such, the overall question this paper addresses is how standardized processes, in conjunction with organizational culture, enable or deter the development of collaborative system thinking within engineering teams.

\section{Motivation}

The US Air Force estimates that greater than 35\% of cost and schedule overruns are attributable to a "lack of adherence to systems engineering practices and principles." (Loren, 2004) Further complicating the situation are programs with increasingly longer development times. Estimates in (Murman et.al., 2002) show an engineer graduating today can expect only one military aircraft development program during his or her career compared to at least five such opportunities for an engineer who graduated in the 1950s. The result is that while current systems engineering practices leave room for improvement, there are fewer and fewer opportunities to apply the processes and learn from experience.

Standardized processes offer an opportunity to codify best practices and facilitate effective coordination among individuals and groups working on a complex problem. The world is full of standards that facilitate communication and ease tasks in daily life: the meaning of green and red street lights, the shape of an electrical outlet, and written language. Yet, anecdotal evidence shows some resistance to standardized processes in engineering. Those opposing standardization cite issues of inflexibility and impeded creativity as their reasons for concern. Schedule and budgetary concerns are also popular reasons for opposing standardized processes. While some processes have been designed in ways that conflict with human nature, (Pajerek, 2000) is quick to point out evidence does exist for the benefits of process improvements.

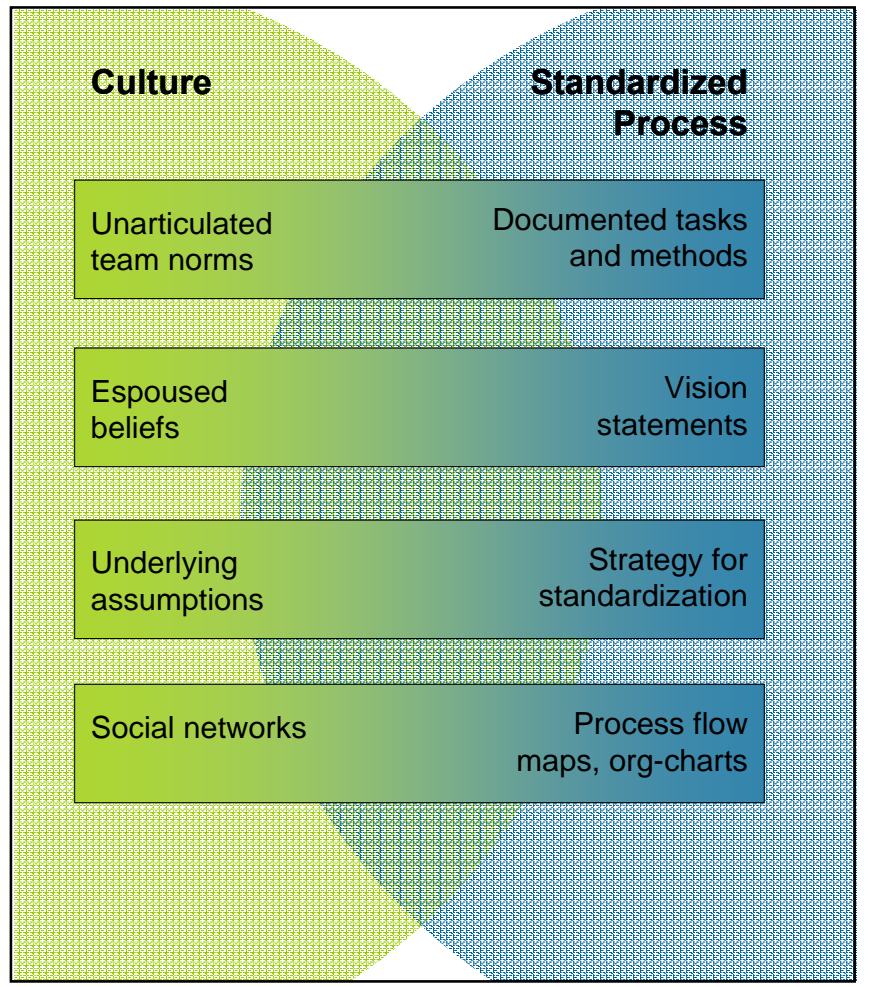

Figure 1. Proposed interfaces between the social and technical subsystems: culture and standardized process. 
In order to better understand standardized processes and their role in organizations, consider the organization as system analogy. Within this analogy, an organization is equated with a large socio-technical system composed of the technical, or process, subsystem and the social, or personnel, subsystem. (Pajerek, 2000) As the technical subsystem, processes can themselves be engineered and improved. By contrast, it is much more difficult to affect meaningful and directed change within the social subsystem. From a systems engineering perspective, interfaces must be well designed and well managed between the social and technical subsystems. Figure 1 proposes a few possible interfaces. Previous research has investigated the development of systems thinking within individual engineers. (Davidz, 2006) This research focuses on the social-technical interfaces as a means of developing collaborative systems thinking within teams of engineers and engineering organizations.

It is hoped that by better understanding the interfaces between the personnel and process subsystems of an organizations that improvements could be made in process design, implementation and maintenance with an emphasis on developing systems thinking within teams. Such changes might move engineers from their current state of grudging process compliance to a state of process commitment. While the compliant engineers follow the process, committed engineers innovate and develop the process, being empowered to both use the process and to evolve the process in light of new lessons learned.

Additional motivation for linking the technical and social views of process can be found in descriptions of the benefits of cultural analysis and systems thinking. Both promise to make sense of complicated situations. Systems thinking, by definition, is a way to look at the whole and understand "interconnections, interactions, and interdependencies." (Davidz, 2006) In his book Organizational Culture and Leadership, (Schein, 2004) offers a strikingly similar explanation for the benefits of cultural analysis. Schein says that "once we learn to see the work through cultural lenses, all kinds of things begin to make sense that initially were mysterious..." As culture is a trait of groups of people, the cultural lens has the added benefit of explicitly considering the effects of teams on the completion of work and serving as a means to extrapolate the concept of systems thinking to teams.

This research focuses on teams, because when designing complex systems, teams are the fundamental work unit of today's organizations. (Hackman, 2002). Modern engineering practices like integrated product teams (IPT's) and integrated concurrent engineering (ICE) are dependent on the ability of teams of individuals to make design decisions and trade-offs at the teams and organization levels. Individuals with systems thinking capability can greatly facilitate team decision making. However, the authors postulate that there are mechanisms operating at levels greater than a single individual which facilitate or hinder the ability of a group to effectively and efficiently undertake design. Standardized processes are likely one such mechanism.

Finally, this work should come under the auspices of engineering study rather than business and management because systems engineering is predicated on analysis at the level of a whole, looking across disciplines. (Rouse, 2005)

\section{Background}

Three key concepts are used to explore existing research and begin to propose interfaces: process, culture, and systems thinking. Figure 2 illustrates the three concepts and the interactions of interest. 


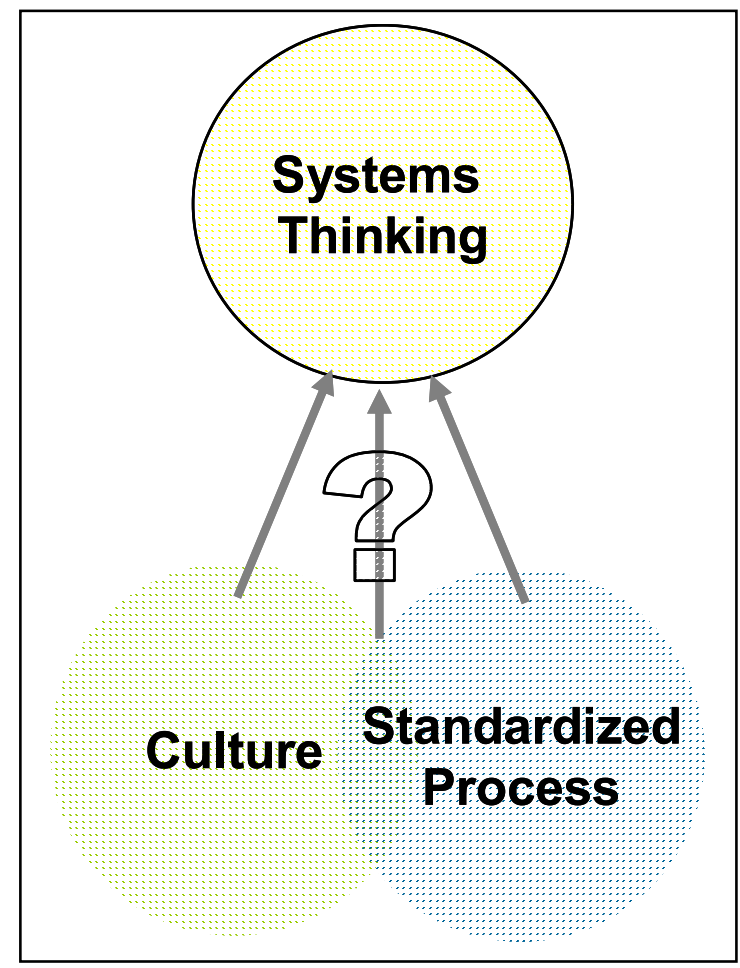

Figure 2. Illustration of three key research constructs and proposed interactions. The arrows and question mark identify the construct interfaces on which this research will focus.

Process. Process is a logical sequence of tasks performed to achieve an objective, a way of decomposing a large task into smaller subtasks. Process defines what is to be done without specifying how. (Martin, 1997) Methods are then used to specify how tasks should be completed, and tools enable or automate a method for completing a process. The systems engineering process spans the lifetime of a product and consists of high level tasks including defining the problem, requirements analysis, functional analysis, design reviews and system operation and life cycle support. (Blanchard, 1998) Processes can take many forms. Processes may be standardized across an organization, agreed upon within a smaller group, or unarticulated sets of common assumptions. (Malone, 2004) Unarticulated processes can include accepted ways of acting in a group meeting, or asking for help and are considered components of culture.

Within engineering, standardized processes are used to decompose large problem approaches into smaller tasks and facilitate collaboration among the teams addressing each task. The following is a discussion of the benefits and drawbacks of standardized processes.

The benefits of standardized process include the promotion of consistent design practices, reducing variability and ambiguity; development of process maps, providing individuals and teams with an understanding of their role in the overall whole; and acting as an experience leveler for younger engineers. Within the Toyota Production System, rigid process specifications are seen as supporting flexibility and creativity. (Spear and Bowen, 1996) Within the scientific method of Toyota's process improvement, rigidly defined processes allow for easy measurement of the benefits of incremental process improvement. The failure to specify standard processes is s systemic inefficiency. In the absence of standard process, new members to an organizations or team must relearn what does or does not work, thus duplicating efforts- 
often referred to as 'reinventing the wheel.'

The proposed limitations of standardized process conflict with many of the espoused benefits. Specifically, some postulate rigid processes reduce overall flexibility. In quickly changing environments, (Malone, 2004) proposes that rigid processes inhibit an organization's ability to improvise and capitalize upon new situations. Malone continues by stating process maps too often specify how work is to be done, rather than identifying the tasks to be completed. It is easy to commit the error of confusing the abstract entity of the process with the actual execution of the process by an individual or group. (Pajerek, 2000) Crossing the process/method barrier may explain why some engineers resist standardization, as work is more rewarding when it includes the latitude to make judgments and choose how to accomplish a task.

Organizations adopt process through a combination of organically grown procedures and by adopting other's best practices. Many organizations evaluate their process maturity through assessments such as the Software Engineering Institute's Capability Maturity Model Integration $\left(\mathrm{CMMI}^{\circledR}\right)$ approach. Such assessment methods recognize the need to tailor processes to the specific organization without explicitly considering an organization's culture. There is still much room for clarification on the correct level of process specification or how process tailoring within an organization should be handled. Some process advocates insist the development process should be tailored to the specific product under development. (Martin, 1997) However, the view of standards as rules of engagement, forming a set of mutual expectations lends to the view that standards should be kept at a higher level, either at the organization or multiorganization levels. Higher-level standards facilitate collaboration among organizations. The paradox of standards is finding a balance between specifying sufficient detail to help everyone move in the same direction while being broad enough to allow collaboration and establish connections between diverse activities. (Malone, 2004) These implications limit our ability to engineer and optimize processes in the same manner as systems engineering is used to design and optimize systems. (Pajerek, 2000)

Culture. Process is not enacted in a vacuum. Rather, process is executed within teams and organizations possessing unique cultures and social structures representing the social subsystem. Culture is defined as a "dynamic phenomenon and a set of structure, routines, and norms that guide and constrain behavior." (Schein, 2004) The social structure includes an organization's formal hierarchy as well as the unspecified networks of knowledge and interactions that develop naturally within organizations and support process execution. Whereas the inherent hierarchy and ability to decompose process facilitates a mechanistic system view, a more organic systems perspective is required to understand an organization's social structure. An organic perspective facilitates viewing an organization as a dynamic, living entity. (Lipnack and Stamps, 1994)

As defined by (Schein, 2004), culture is composed of three components: norms of behavior, espoused beliefs, and basic underlying assumptions. Intangible components, such as relationships, interactions, and behavioral norms are unarticulated characteristics of teams and organizations. The intangibles support how work is accomplished. By forming a set of shared assumptions and accepted behaviors, cultural norms likely support collaborative systems thinking. This explains, in part, why team performance is higher when membership is stable. However, because culture amounts to a set of unwritten standards, the intangible aspects of culture likely form barriers to teams with dynamic members developing systems thinking capabilities.

Another property of social systems is the path dependency of information. The fluid nature of information within a social network is a barrier to collaborative systems thinking because 
gathering accurate and objective data necessary to understand the 'big picture' is more difficult. Tangible components of culture and social structure, such as organizational charts, job titles, and IPTs form visible artifacts that explain how an organization is arranged and operates. Rotation programs, communication forums, and communities of practice are tangible structural elements that may facilitate higher level systems thinking.

Interfaces. To continue the organization as system analogy, interfaces exist between the social and technical subsystems. One such interface is illustrated by the failure of proven processes to guarantee success as demonstrated by three examples.

First is the failure of others in the automotive industry to successfully implement the Toyota Production System even though these processes are well documented. (Spear and Bowen, 1999) This failure points to a misalignment between standardized process and an organization's basic underlying assumptions. The second example comes from a study observing product development teams. In her study of organizations developing models for new product markets, (Dougherty, 1990) noted successful developers were more likely to interact outside of and beyond those interactions specified by standard process. These groups challenged each other, developed organic relationships and challenged established ways of working. (Dougherty, 1990) Furthermore, the new processes developed were not documented and retained, that is to say the successful teams did not create or modify the standard way of operating based on lessons learned through their successes. The success of those teams interacting beyond standardized process shows the role of team norms in enhancing performance. The final example reinforces the role of team norms as a compliment to standardized process. Referenced in (Hackman, 2002), a study by Martine Haas showed that teams allowed to develop their own strategies for dealing with information rich environments faired better than teams bound by predetermined processes. Given engineering environments are data rich, this study calls into question the efficacy of standardized processes in helping groups cope with making decisions based on large quantities of data.

Other interfaces likely exists between the social structure-knowledge and interaction networks - and standardized process. Explicitly considering these interfaces when tailoring process will allow for new insights into the benefits and drawbacks of standardized processes. For example, a rigid interface would allow individuals to move freely within an organization without the need to relearn process. In contrast, a flexible interface might allow for individual teams to tailor process, or even develop their own processes.

A common saying is "you are what you measure." A possible corollary is "you become what you measure." In an environment where metrics are religiously collected and used to gauge the strength and progress of teams and organizations, insight into the interactions of process and culture will help to design metrics that will lead teams and organizations to evolve in ways that better align process and culture.

Systems Thinking. The third concept of interest is systems thinking. Having analyzed several divergent definitions for systems thinking (Davidz, 2006) developed a definition for systems thinking as the "analysis, synthesis, and understanding of interconnections, interactions, and interdependencies that are technical, social, temporal, and multi-level." Author of The Fifth Discipline, (Senge, 2006), defines systems thinking as the tools and accompanying body of knowledge developed to make "full patterns clearer and to help us see how to change them effectively." Davidz's definition was developed to characterize the systems thinking of individual engineers. Senge's definition, while defined for organizations, is too broad for characterizing the unique aspects of systems thinking in engineering. Other, notably (Frank, 
2000), have looked into tailoring Senge's laws of systems thinking for engineering, but have not yet made the transition to looking at systems thinking as a property of a team of engineers. For products of great complexity, teams are the functional unit for executing design. As such, it is appropriate to explore the concept of systems thinking within engineering teams; that is, to define what traits and activities within a team or organization constitute collaborative systems thinking.

Children learn systems thinking quickly, however, children are taught at an early age to solve problems by decomposition, in the process loosing a sense of the larger whole. (Senge, 2006) Standardized processes are themselves a means to decompose a large design problem into smaller parts that can be individually solved. Much as the child looses sight of the larger whole over time, so too might standardized processes function as a mask, preventing engineers from seeing the overall design problem. Loosing sight of overall whole may lead teams to broker inefficient tradeoffs, compromising some aspect of the final system.

Mental models offer one exploration of how standardized process may promote systems thinking. Mental models affect how individuals interact with the environment and make decisions. (Newman, 1999) Much like engineering models, mental models are simplifications of reality based on truths, part-truths, and missing or implied information. Documented standardized processes may form the basis for mental models of the development process. Because mental models influence the ways individuals interact with their environments, an accurate mental model of the development process is necessary to facilitate efficient and effective decision making. However, a mental model is specific to an individual. Engineering is a socio-technical activity requiring many people to interact. If each individual has a slightly different mental model of the development processes, unarticulated assumptions may cloud their collective ability to execute the process.

Shared mental models - shared views of reality that develop within a group over time-offer a mechanism to frame systems thinking as a team characteristic. Shared mental models may explain why stable groups work more efficiently together. Formal and informal reviews, by emphasizing requirements and surfacing assumptions, may help to form shared mental models thus facilitating systems thinking.

Systems thinking within teams is embedded at the intersection of how process is specified and how work is actually done. To paraphrase former vice president of Bell Labs, Estill I. Green, 'groups do not create ideas, individuals do.' (Martin, 1997) While this is true, it is through communicating ideas that design is accomplished. Design is a dynamic process and sometimes the problems encountered are a result of static views rather than process thinking. (Senge, 2006) Process thinking and the sharing of mental models are likely enablers of collaborative level systems thinking. However, the process by which teams develop shared

mental models is unclear. Perhaps standardized processes form a set of shared assumptions and facilitate the formation of shared mental models.

\section{Research Design}

Exploratory Research. This ongoing research is an exploratory study of the ways in which standardized processes and organizational culture enable or deter collaborative systems thinking within teams of engineers. Whereas traditional deductive research starts with a hypothesis and then seeks evidence to prove or disprove the hypothesis, exploratory research starts with an interesting question or area of inquiry and ends with a set of hypotheses that form the basis for new theory. (Glaser and Strauss, 1967) This is known as grounded theory research. Grounded 
theory research is characterized by concurrent and systematic data collection, analysis, and theory development. (Glaser and Strauss, 1967, Strauss and Corbin, 1998) Because grounded theory research utilizes a systematic process to collect and analyze data, it leads to a more accurate process of discovery.

Case studies will be used to populate the data set. Case studies are well suited for exploratory research because they are a flexible and effective means to gather many types of information. (Yin, 2003) In addition, case studies are helpful in establishing external validity of the data collected as well as increasing the generality of findings. (Yin, 2003)

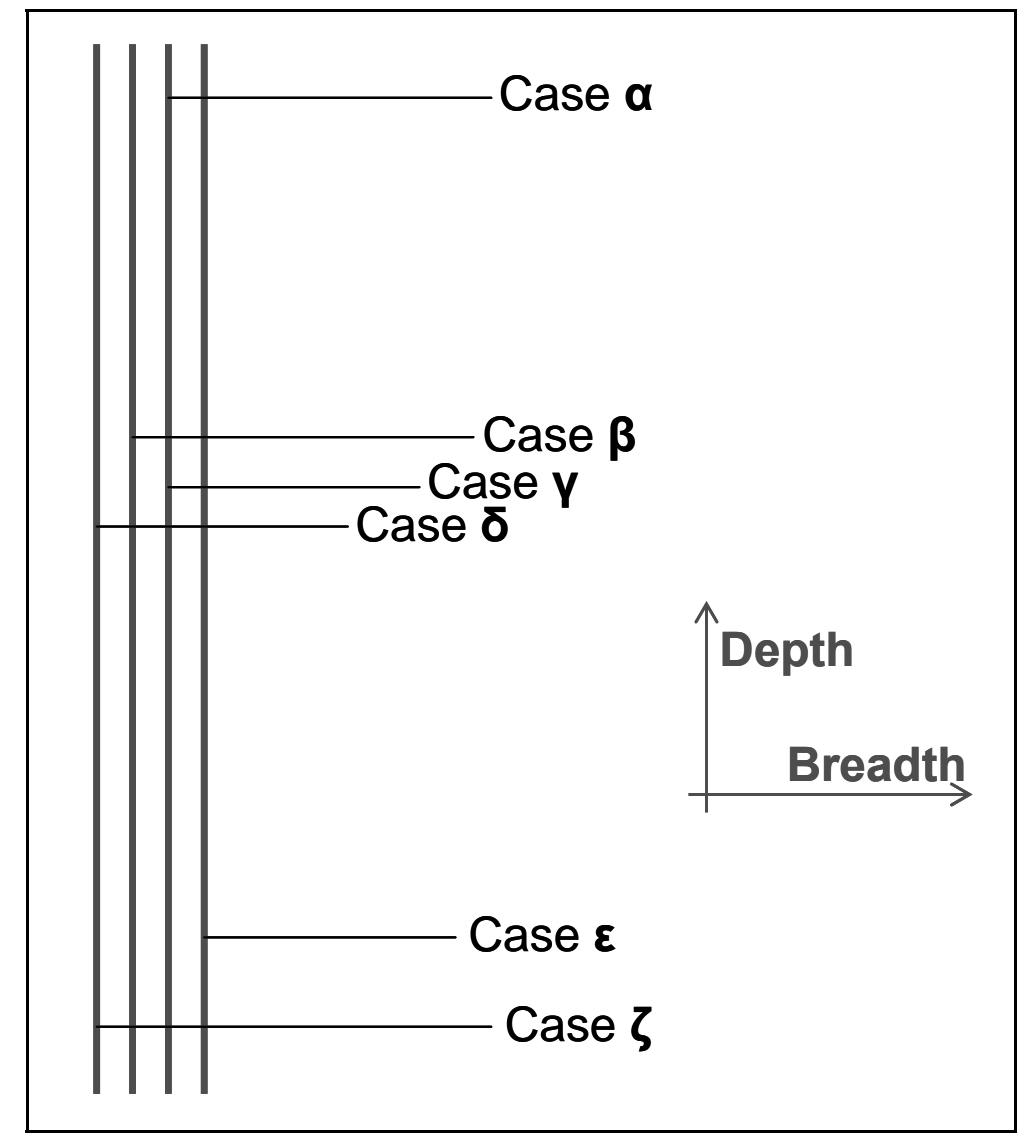

Figure 3. One in-depth case study will be supplemented with several smaller case studies to provide breadth to the research.

Case Study Selection. As stated above, this research will involve several case studies. As a general rule of thumb six to ten cases are necessary to achieve convergence. (Yin, 2003) Companies in the aerospace sector will be preferentially selected due to the first author's aerospace background and association with the Lean Aerospace Initiative (LAI), a research consortium headquartered at the Massachusetts Institute of Technology.

Figure 3 shows the proposed case study structure. One in-depth case study will be supplemented with several smaller case studies to increase the generality of results. The in-depth case study is at the time of publication already selected and follows an organization as it updates its standardized process set, allowing for time series data collection to ensure the research results are generalizable. Additional, smaller cases will be selected from organizations with varying 
levels of process maturity.

Within each case study, interviews, surveys, and primary process documentation will be the primary sources of data. By combining data from multiple sources, a richer picture will emerge of how the engineering process is specified and applied, and how it interacts with culture.

Pilot Interviews. Initial inquiry will take the form of pilot interviews. In these interviews heads of organizations and established systems engineers will be asked questions aimed at clarifying and defining the key research constructs. All interviewees will be provided with a copy of Davidz's definition for systems thinking, and the following are examples of questions:

1) How might the definition for systems thinking change when perceived as a property of a team of engineers rather than as single engineer?

2) Have you participated in a group you would describe as possessing systems thinking characteristics? What steps were taken within this group to promote systems thinking?

3) What enablers, barriers and/or precursors exist to the development of collaborative systems thinking?

4) Must individual team members express systems thinking capabilities in order for a team to possess collaborative systems thinking characteristics?

The answers obtained during pilot interviews will be used to inform the format of interviews and survey instruments to be used during the case study portion of this research.

Case Study Design. After results from the pilot interviews are analyzed, case studies will be selected, designed, and executed. Within each case, multiple levels of analysis will be considered. Individual interviews, surveys, and focus groups will all play a role in gathering data. The following provides the concepts for what types of data each method will collect.

Surveys will be used as a tool to define the structural aspects of an organization. Primarily social network data will be gathered: information to populate knowledge and interaction networks. Examples of social network data include patterns of interaction (exchange analysis), clustering and differences between an organizations formal structure and underlying social structure. (Allee, 2003) Additional questions will gauge team stability, team identity, and patterns of process use within teams.

Interviews are a good method for asking open-ended questions. As such, interviews will address issues of culture, process compliance, and identifying areas where improvement is needed. The role of process in culture formation and change, the nature of standards usage (checklist vs. informative prescriptive tool), and the role of standards in promoting mental models of the development process are all potential areas of inquiry. Additional questions will address the impact of process standardization and the resulting artifacts on systems thinking.

Focus groups will be used as a tool to observe team interaction for the purposes of calibrating individual interview data. Focus groups are also an opportunity to collect team perspectives of process design, use and maintenance. Team norms and the impact of flexible team membership can also be addressed in focus groups.

Additional data will come from primary resources such as organizational charts, process flow charts, and process documentation. Triangulation of sources will be used to bolster internal validity of findings. External validity will be addressed by selecting a diverse set of cases.

\section{Conclusion}

As of this paper's publication, the research described herein has been ongoing for about six months. Building on research on the enablers and barriers to systems thinking at the individual 
level, this paper provides an overview of the motivation for studying the role of standardized processes and culture as enablers or barriers to systems thinking at the team and organizational levels. The research design outlined is an exploratory, case-study based plan using interviews and surveys to gather data. Outputs of this research will include an operational definition of collaborative systems thinking, identification of the ways in which standardized processes act as enablers or barriers for higher level systems thinking, and a set of heuristics for better implementing standardized processes to capitalize on enablers of collaborative systems thinking and limit the impact of identified barriers. These results should help organizations better design, implement, and manage standardized processes that facilitate true learning and understanding in engineering organizations. Secondary benefits might include improved information sharing among individuals and teams, reductions in development times, and a shift from process compliance to process commitment among employees and teams.

\section{References}

Allee, Verna, The Future of Knowledge. Butterworth-Heinemann, Boston, 2003.

Blanchard, Benjamin, Systems Engineering Management. John Wiley \& Sons, Inc., New York, 1998.

Davidz, Heidi, Enabling Systems Thinking to Accelerate the Development of Senior Systems Engineers, Doctor of Philosophy in Engineering, Massachusetts Institute of Technology, Cambridge, 2006.

Dougherty, Deborah, "Understanding New Markets for New Products", Strategic Management Journal, 11, 1990, 59-78.

Frank, Moti, "Engineering Systems Thinking and Systems Thinking”, Systems Engineering, 3:3. 2000, 163-168.

Glaser, B., and Strauss, A., The Discovery of Grounded Theory. Aldine Publishing Company, Chicago, 1967.

Hackman, J. Richard, Leading Teams. Harvard Business School Press, Boston, 2002.

Harris, David, "Supporting Human Communication in Network-Based Systems Engineering", Systems Engineering, 4:3, 2001, 213-221.

Johnson, Stephen, "Three Approaches to Big Technology", Technology and Culture, 38:4, 1997, 891-919.

Lemos, Robert, "NASA Fights Premature Graying", Wired News, http://www.wired.com/news/technology/space/0,71822-0.html, (Accessed December, 2006).

Lipnack, J. and Stamps, J., The Age of the Network. John Wiley \& Sons, Inc., New York, 1994.

Loren, J.R., USAF Systems Engineering-Revitalizing Fundamental Processes, Technical Proceedings of 2004 USAF Developmental Test and Evaluation Summit (Woodland Hills, CA, 16-18 November, 2004).

Malone, Thomas, The Future of Work. Harvard Business School Press, Boston, 2004.

Martin, James, Systems Engineering Guidebook. CRC Press, Boca Raton, 1997.

Murman, E., et.al., "Challenges in the Better, Faster, Cheaper Era of Aeronautical Design, Engineering and Manufacturing." Technical Report RP00-02, Lean Aerospace Initiative, September 2000.

Newman, Richard, "Issues in Defining Human Roles and Interactions in Systems", Systems Engineering, 2:3, 1999 143-155.

Murman, E., et.al., Lean Enterprise Value. Palgrave, New York, 2002.

Pajerek, Lorraine, "Processes and Organizations as Systems", Systems Engineering, 3:2, 2000, 
103-111.

Rouse, William, “A Theory of Enterprise Transformation”, Systems Engineering, 8:4, 2005, 279295.

Schein, Edgar, Organizational Culture and Leadership. Jossey-Bass, San Francisco, 2004.

Senge, Peter, The Fifth Discipline. Double-Day, New York, 2006.

Spear, S. and Bowen, H.K., "Decoding the DNA of the Toyota Production System", Harvard Business Review, Sept-Oct, 1999, 96-106.

Strauss, A., and Corbin, J., Basics of Qualitative Research. Sage Publications, Thousand Oaks, 1998.

Yin, Robert, Case Study Research Design and Methods. Sage Publications, Thousand Oaks, 2003.

\section{Biographies}

Caroline Twomey Lamb is a doctoral research assistant at MIT's Lean Aerospace Initiative (LAI). She is pursuing her degree through the MIT Department of Aeronautics and Astronautics. Her primary interests are in the aerospace industry. Past research includes modeling and analyzing turbine quality control procedures and participating in a study to quantify previously un-calibrated turbulence in MIT's Wright Brother's Wind Tunnel. Current research concentrates on the aerospace industry at the enterprise level. Ms. Lamb received her S.B and S.M from MIT in 2003 and 2005 respectively and currently serves as the student liaison to the board of directors of the American Institute of Aeronautics and Astronautics (AIAA).

Dr. Donna H. Rhodes is a past president and fellow of INCOSE, and a recipient of the INCOSE Founders Award. She is Senior Lecturer at MIT's Engineering Systems Division (ESD) and a Principal Research Engineer at MIT's Center for Technology, Policy, and Industrial Development. She is the Director for the MIT Systems Engineering Advancement Research Initiative (SEARI). Her areas of specialization include technical and management theory and practices for architecting and design of complex systems, systems-of-systems, and enterprises. Dr. Rhodes has 20 years of experience in the aerospace, defense systems, systems integration and commercial product industries. She serves on a number of corporate, university, and government boards focused on the advancement of the systems practice and education. 